\title{
Hypoglycemic Detection by Human Breath: A Mobile Health App that Alerts Diabetics of Low Blood Glucose
}

\author{
A. Faiola ${ }^{1, *}$, H. Vatani ${ }^{1}$ and M. Agarwal ${ }^{2}$ \\ ${ }^{1}$ Dept. of Biomedical \& Health Information Sciences, University of Illinois at Chicago, Chicago, IL, USA \\ ${ }^{2}$ Dept. of Mechanical Engineering, Purdue University, IUPUI, Indianapolis, IN, USA
}

\section{Abstract}

Low blood glucose (BG) or hypoglycemia (HYPO) can lead to severe health complications such as weakness and unconsciousness. To avoid problems BG self-management is needed. We developed a non-invasive breathing system (HYPOalert) to detect HYPO in human-breath, that sends warning alerts and data visualization to monitor progress. This paper presents two HYPOalert prototype iterations with testing results. Of 14 Type $1 / 2$ diabetics tested, only $10 \%$ were pleased with existing monitoring systems and $85 \%$ expressed interest in using HYPOalert more than 20x a day. The usability study showed that $92 \%$ agreed-strongly agreed with the HYPOalert design, including color/menus/navigation/ typography; and $64 \%$ felt positive about the apps consistency, flexibility, and info architecture. A post-test survey provided a satisfaction score: 6.64/10, with an open-ended interview showing that HYPOalert could positively impact lifestyle practices, self-managing, and help advance an understanding of the disease.

Keywords: Hypoglycemia, diabetes, breathing sensor, mobile health, interface design, data visualization

Received on 10 October 2018, accepted on 21 January 2019, published on 12 December 2019

Copyright (C) 2019 A. Faiola et al., licensed to EAI. This is an open access article distributed under the terms of the Creative Commons Attribution licence (http://creativecommons.org/licenses/by/3.0/), which permits unlimited use, distribution and reproduction in any medium so long as the original work is properly cited.

doi: 10.4108/eai.23-3-2018.162220

\section{Introduction}

In 2012, the American Diabetes Association stated that one-tenth of all health care dollars were spent on costs directly attributable to diabetes - the total cost: \$245 billion. More importantly, over half of this cost was directly or indirectly resulting from poor maintenance of blood glucose (BG) levels [3]. Diabetes has two classifications with accompanying medical conditions-depending upon the glucose level. Type 1 diabetes (T1D) is characterized by deficient insulin production and requires external insulin administration every day, and Type 2 diabetes (T2D) is an outcome of the body's ineffective usage of insulin [6]. The direct healthcare costs per-person for Type 1 diabetes (T1D) are 50\% higher than for Type 2 diabetes (T2D) [1]. According to recent data, there are more than $970 \mathrm{~K}$ people living in the US who have T1D, with projections that by 2050, more than half of these will be children. Diabetes mellitus is a chronic disease caused by the body's insufficient production of insulin. As a hormone, insulin

*Corresponding author. Email:faiola@uic.edu regulates $\mathrm{BG}$ levels, thus diabetics take insulin as an external medication to regulate BG levels [2].

Managing diabetes requires tight control of BG levels, where too high BG results in hyperglycemia (HYPER) and causes long-term complications. Conversely, low BG results in hypoglycemia (HYPO, i.e., BG levels of $<70$ $\mathrm{mg} / \mathrm{dL}$ ) [4] and can cause short-term complications including unconsciousness or permanent brain damage. Severe complications can lead to death. HYPO, common in T1D, is most dangerous for infants, young children [16], elderly [10]. Individuals with diabetes have differing ability to sense their HYPO symptoms, and some can entirely lose the ability over time, a condition called hypoglycemia unawareness [8]. In addition, the fear of the impaired cognition and more dramatic symptoms of HYPO causes many patients to err towards HYPER and lead passive lifestyles, including decisions not to engage in healthy exercise [5] and not driving [14].

Of the mHealth diabetes devices and applications (apps) on the market, there are four basic ways of glucose monitoring: skin prick, saliva, urine, and continuous 
glucose monitoring (CGM) systems. The most used methods for detecting HYPO are invasive and rely on analyzing glucose levels in blood (through a lancet fingerstick) or interstitial fluids (through a subcutaneous injection of a CGM) [9]. However, CGMs often under-report HYPO [7] or display a significant time lag before indicating HYPO [11]. For an individual with hypoglycemia unawareness, this can lead to a dangerously low BG before it is treated. A recent study showed CGMs may not significantly improve glycemic control in children with T1D [12]. Each method has its own limitations related to invasiveness, expense, and availability. There is, however, a method that has shown considerable promise is trained canines - diabetes alert dogs (DADs), that act as an early warning system by alerting patients without any noticeable symptoms of HYPO [15] [16].

As noted, research has demonstrated that DADs can smell the metabolic by-products of HYPO even before it registers on a glucose-based detection device, prompting patients to check their BG levels and take action before they suffer a HYPO episode. This early detection is due to the ability of DADs to smell volatile organic compounds (VOCs) from breath caused by metabolic changes that lead to HYPO, not by smelling glucose itself. Preliminary studies indicate patients with DADs have improved glycemic control and improved quality of life with fewer complications and lower healthcare costs. However, there are long wait-lists and many costs associated with training and maintaining DADs. As such, we believe an accurate, affordable, and non-invasive device can be designed to detect (through human breath) these metabolic changes. The smart sensor system will mimic the DAD's ability to detect HYPO from VOCs in breath-thus being a technological breakthrough in hypoglycemic detection, while decreasing costs of care, decreasing morbidity and mortality, and transforming the health and well-being of patients with diabetes.

\section{Proposed Research}

In response to these challenges, we are in the final phase of designing and developing a non-invasive connected mobile health system for T1D and T2D patients - that includes a canine-inspired, smart nanosensor (breathing device) technology that connects via Bluetooth with a mobile app. We tentatively refer to the system as: HYPOalert. The app has the capability of $24 / 7$ (HIPAA compliant) determination of HYPO, while providing early warning alerts detecting chemical changes in human breath. Incorporated into a smart device, the sensor system will communicate health data to caregivers, collect data for proactive HYPO treatment, and integrate with other diabetes management devices. See Figure 1.

Thus far, the research team has collected and analysed human breath samples using gas chromatography/mass spectrometry - creating signature VOC breath profiles that correlate to HYPO. Second, the design, fabrication and testing of the nanosensor array and breathing smart sensor device will allow patients to obtain a registered responsearising from any detected metabolic change during a transient condition leading to HYPO. Each sensor on the array will respond to one or more of the identified VOCs. The sensor array will be equipped with computational resources and wireless data transmission-communication modules. Data will be transmitted via Bluetooth and mobile devices (e.g., smartphone) — alerting patients or caregivers (between doctor-recommended blood glucose measurement intervals) that the patient may be trending towards HYPO and needs to test $\mathrm{BG}$ levels or take immediate corrective action.

The focus of this paper outlines the design and testing of the first two prototype iterations of the mobile app - that will accompany the forthcoming smart breath device system.

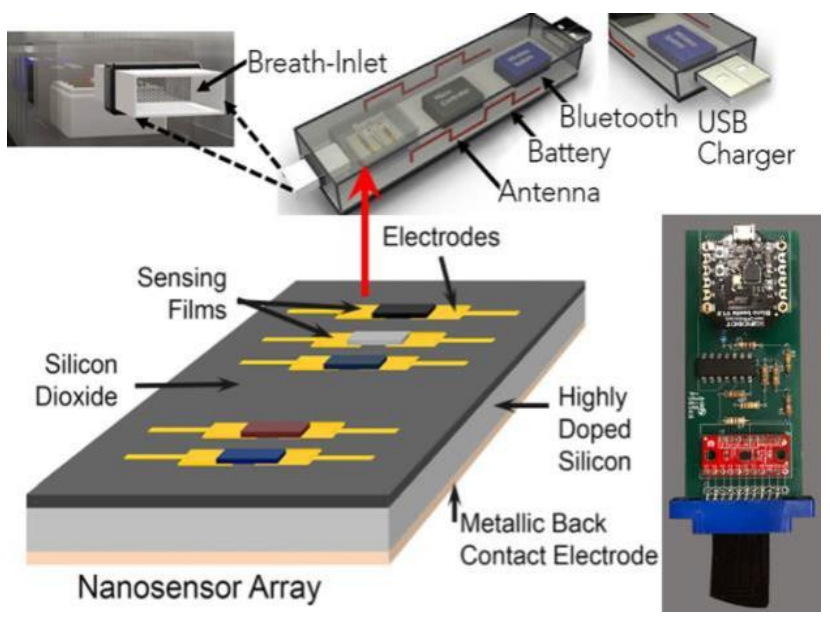

Figure 1. Illustrates the nanosensor array and device configuration with breath-inlet, antenna, battery, Bluetooth, and charger.

\section{Design Iteration-Phase 1}

\subsection{Design Process}

Based on a preliminary review of existing diabetes products and mobile apps, we developed a series of exploratory static/rapid interfaces for inspection by diabetes patients. As outlined, the primary purpose of HYPOalert is to warn patients of an approaching or existing state of HYPO. For this reason, the initial interfaces reflect this primary functional aspect. Figure 2 illustrates the first conceptual iteration of HYPOalert.

Based on this iteration of interfaces, we performed a user requirements study-focusing on understanding user needs through questionnaire, semi-structured interviews, and a preliminary review of the first iteration of the mobile app interface design. Findings provided design requirements and the users' overall perception and interest in a breath smart sensor app for supporting HYPO monitoring. 

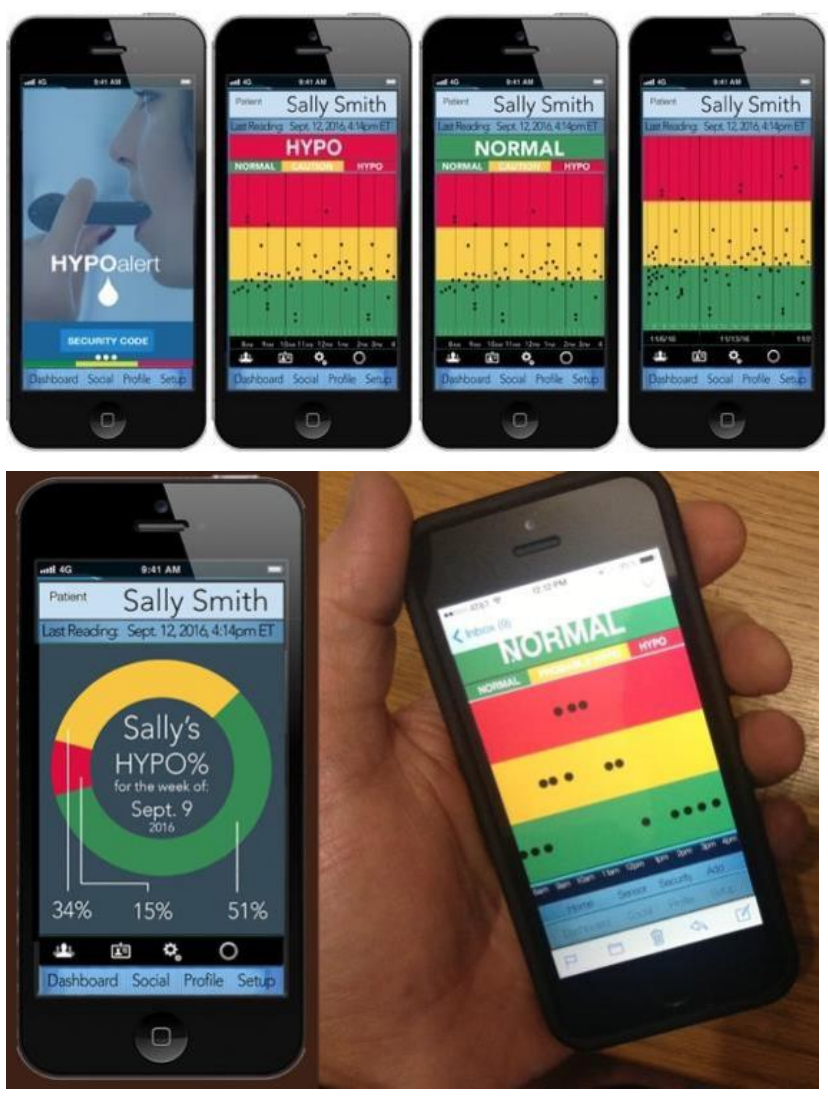

Figure 2. First iteration of the HYPOalert static interfaces. First interface displays the app splash-page, with remaining interfaces indicating the patient's name and reading date/time. Interfaces 2-3: (1) Three colorcoded visualization areas: Green=NORMAL, Yellow=APPROACHING (or Caution), and Red=HYPO, (2) Hourly indication of breathing times and their location on the HYPO visualization scale, and (3) breathing times over several months. On the bottom: Interface $(L)$ display breakdown of the patient's weekly breathing summary in percentages; and last interface (R) demonstrates HYPOalert on smartphone in hand.

\subsection{Methods}

Participants: We recruited 20 participants with Type 1 and Type 2 diabetes (12 T1D / 60\%; 8 T2D / 40\%), $n=20$. Recruitment included paper flyers that were circulated throughout the University of Illinois at Chicago (UIC) campus and a bulk email sent through the UIC listserv system to all UIC staff and students. All participants received an Amazon $\$ 10$ eGift for their participation, sent via email. Inclusion criteria included: volunteer's age must be above 18 years, must speak fluent English, and must have Type 1 or 2 diabetes. All vulnerable populations were omitted.

Interview, Questionnaire and Rapid Prototype Review: All participants received eight open-ended interview questions and 13 multiple-choice survey questions. At the conclusion of the questionnaire, participants were shown the first iteration prototype of the mobile app interface design of HYPOalert. After a short time to review the interfaces, all participants were given follow-up questions related to the product's benefits, functionality, and overall appeal.

Data Analysis: The results from 13 survey questions were analyzed by calculating the mean and standard deviations (SD) - converting integer values using Excel 2010. Using histogram values, each question was displayed as bar plots and pie charts graphics.

\subsection{Findings}

Questionnaire: Questionnaire results regarding the method for tracking BG levels showed that $65 \%$ participants use the skin prick, $30 \%$ used both continuous glucose monitoring and skin prick, and 5\% used urine testing. Regarding HYPO management, 35\% of participants had sugar intake at random hours, $25 \%$ make use of health apps to track glucose levels by measuring food intake and physical exercise, while only one participant uses noninvasive glucose tracking. Only three participants use a combination of all three methods for managing hypoglycemic levels. Only $20 \%$ of participants did not use any method to track their hypoglycemic levels.

With regard to levels of satisfaction with current monitoring systems, $10 \%$ of participants were pleased with their current system, $45 \%$ participants were somewhat satisfied, and $40 \%$ were not satisfied with their current BG measuring method or devices. A total of $85 \%$ of participants expressed their interest to use the proposed HYPOalert breathing sensor over their current HYPO management device. Regarding the extent of use of a breathing sensor, $70 \%$ wished to use HYPOalert more than 20 times a day, if available-while only $20 \%$ expressed that they would only use it once a day to manage their HYPO condition.

Regarding preferred BG data display types, $40 \%$ of participants wanted to see their data visualized along with alerts, rather than a color-coded visualization. At the same time, $45 \%$ wanted to see the data visualization color-coded, with numbers, color coding data, and line/bar plots - similar to the HYPOalert app interface. Finally, $15 \%$ expressed an interest in only visualizing their BG data with bar or line plots. And, regarding sharing their data with others, 95\% stated their interest in including their family, while $90 \%$ wanted to include a means to share their HYPO data with their primary provider.

All participants were interested in using the HYPOalert app, with its data visualization systemstating that it would help advance their understanding and management of their HYPO condition. All participants $(100 \%)$ were slightly to strongly in agreement that the proposed HYPOalert system (sensor and app) would positively impact their daily lifestyle practices.

Open-Ended Interview: The results of the interviews demonstrated that the frequency of both severe HYPO 
and mild HYPO episodes among the participants. We found that the occurrence of mild HYPO episodes (5.1 \pm 7.3 times per week) was approximately 10-fold higher than the severe HYPO episodes $(2.1 \pm 2.3$ times per month). The average participants monitoring of Blood Glucose (BG) levels was $2.8 \pm 2.9$ times per daywhile BG monitoring still varied from patient to patient. For example, one T1D participant monitors BG levels 10 times a day, whereas four participants check their BG randomly throughout the week.

Approximately $80 \%$ of participants use BG measuring devices to specifically manage their hypoglycemia. Although most participants use traditional or advanced BG monitoring devices, the adoption of mHealth apps is very poor, i.e., $75 \%$ of participants do not use any type of mobile app that links their BG results from the device to their smartphone. The remaining 25\% use GoMeal, Dexom, OneDrop, or other apps to review BG data from their monitoring devices.

Regarding the use of HYPO data visualization, the majority of participants $(70 \%)$ recommended that the device saves the breathing sensor data through a log option (up to six months), with the ability to visualize the data at any point in time according to glucose levels, $\mathrm{mg} / \mathrm{dL}$, and trends. In addition, three participants expressed their interest to include an option for food intake data; whereas one participant suggested we include an insulin dosage option that alerts when HYPO occurs. Two patients would like to include predictions or causes of HYPO.

\section{Design Iteration-Phase 2}

\subsection{Product Review}

To obtain a more precise understand of the advancements of other mHealth diabetes products, we conducted an system and interface design audit of two FDA approved mobile products that include both a BG measuring device, with data transmission capabilities (via Bluetooth or directly) to a smartphone app. Our assessment included the Dario and iHealth wireless blood glucose monitoring systems. After registering online, our team tested and assessed the products for two weeks; including daily blood samples. We then de-constructed and built an interface/system information architecture flow-chart, followed by a comparative analysis of the two products using the same criteria.

In sum, both apps offer innovative solutions that improve patient satisfaction by enabling them to manage health more efficiently. Patients can set thresholds for normal and danger glucose levels, personalized goals, medication reminders, diet, and exercise plans. Both products allow users to log their blood sugar level manually, using the device as often as they want. They can also follow their progress visually, and export results as an Excel, PDF, or CSV file to their healthcare provider or family members using e-mail, text, or via shared access in social media.

Our findings showed also showed that there was a lack of consistency in color codes when displaying some of the HYPO BG reading levels, creating some confusion in interpreting the results. For example, although iHealth had a well-designed interface and visualization system, the color-coding system was difficult to distinguish. While Dario provided access to most options with an inside menu, iHealth features and commands can be accessed directly from the interface icons, text, or a combination of both. Unlike Dario, iHealth requires Bluetooth connection, and does not support the visually impaired. However, Dario supports adding emergency contacts and uses GPS to locate the patient when BG levels reach a dangerous range.

\subsection{Design Process}

Findings from the first iteration and the review of the two BG monitoring systems informed our design of the phase two interactive prototype (Figure 3).

\subsection{Methods}

Participants: Inclusion criteria for study two included: participants had to be a graduate student or staff of the University of Illinois at Chicago (UIC), must be 18 years or older, and must speak fluent English. Recruitment was done by circulating invitations for the study across the college via emails, and all vulnerable populations were omitted. The final sample included 14 volunteers from UIC students (78\%) and staff (22\%). The majority of participants were females (71\%), and participants age ranged from 18 to more than 50 years old (average age 34 years). All participants received an Amazon $\$ 10$ eGift for their participation.

Usability Testing: A three-part usability test was done on the interactive prototype (on an iPhone 7 Plus). First, a scenario task-based time-on-task study was completed, that included five tasks related to: 1) registration, 2) setting goal, 3) logging measurements, 4) viewing results and trends, and 5) sharing the results of that day's readings with another person. All participants were observed, and voice recordings were conducting for all task exercises. Second, a post-test questionnaire that included 35 questions was completed by the participants. Twenty-nine questions were grouped in seven categories pertaining to design, functionality, navigation, architecture, benefits, overall appeal of the prototype, and demographics. The participants answered the questions, using a 6-point Likert scale, with 1, signifying strongly disagree to 6 , signifying strongly agree. In addition, there were five questions related to the participants' demographics and experience with mobile phones. Also, there was one question related to the overall total score for user's satisfaction and experience with the HYPOalert 
mobile app-using a 10-point scale. Finally, we asked seven open-ended post-test interview questions related to the participants' experience using the HYPOalert prototype. The intent was to obtain an in-depth understanding of individual experience, perception, and motivating factors for using HYPOalert, followed by any final recommendations.

Data Analysis: The amount of time participants spent to complete each task was measured using descriptive analysis to calculate min, max and average time spent on each task, as well as the whole test. Users' responses during the think aloud process were captured and a thematic content analysis was employed to analyze the data. The responses were organized according to the theme of issues raised during the test to calculate percentage of users who reported the same issues. Survey results for the 35 questions were analyzed by calculating frequency (within the six-point Likert scale-from strongly disagree to strongly agree, with conversion percentages using Excel 2010. We first averaged the scores of each question, according to the responses from the 14 participants. This was followed by averaging those frequency scores that pertained specifically to each focus area of the questions shown in Table 1.

Table 1. Survey focus areas and number of questions.

\begin{tabular}{|l|l|}
\hline FOCUS AREA OF QUESTIONS & \# OF QUES. \\
\hline User Interface & 6 \\
\hline Design Consistency and Flexibility & 6 \\
\hline Navigation and Information Architecture & 9 \\
\hline App Learnability & 8 \\
\hline Overall Score & 1 \\
\hline Usage and Demographics & 5 \\
\hline
\end{tabular}

As such, we could determine to what degree or percentage the overall response was within the range of responses from strongly disagree to strongly agree. For example, under group one, "User Interface Design," there were 6 questions that provided frequencies, with a total overall score of $62 \%$. This frequency score conveyed the degree to which the participants agreed or strongly agreed (liked) the prototype user interface design.

For the interview results, thematic content analysis of the narrative responses was used. The text narrative responses were organized according to concepts and summarized into categorical statements in a spreadsheet to identify key concepts. Confidentiality of the participants was maintained by deidentifying the data and aggregating it into an Excel file for data analysis.

\subsection{Findings}

Usability Testing: Analysis of the results from observing the participants and thematic analysis of their responses during task implementation, demonstrated that the most prevalent usability issues reported by $93 \%$ of users were manual entry of BG into HYPOalert system and interpreting the data visualizations on the logbook. Most users preferred to have the layout of logbook customizable, with clearer actions for selecting the commands on the screen. The second issue reported by $86 \%$ of participants were challenges using the menu options to navigate between different sections of the system. Most users preferred commands as buttons displayed on the interface, rather than hidden inside the menus. More than $50 \%$ of participants commented on clarity of the visuals and display of the legend, colors used, and use of pie charts for displaying the percentages of monthly low, normal and high readings. The average time to complete all 5 tasks and their corresponding subtasks was 11.2 minutes (MIN: 6.5 mins, MAX: 26 mins).

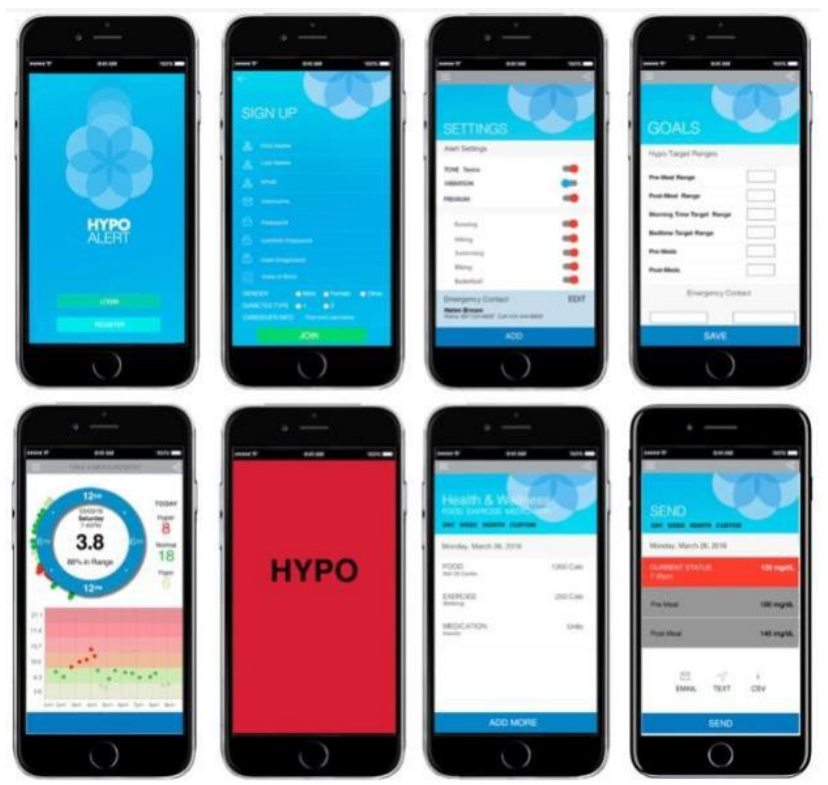

Figure 3. Illustrates the second iteration HYPOalert interactive prototype that focus on data monitoring using several temporal systems, the alert system, along with reminders, settings, and summary reports.

Questionnaire: While $90.9 \%$ of the participants somewhat agreed, agreed, and strongly agreed that the interface design was of high quality related to aesthetics, visual clarity, and meaning, only $60.6 \%$ agreed and strong agreed. In the color palette category, $100 \%$ somewhat agreed, agreed, and strongly agreed that the interfaces, and other visuals and text were highly appealing. While $68.1 \%$ somewhat agreed, agreed, and strongly agreed that the interface design reflected high quality in its design consistency, only $42.4 \%$ agreed and strongly agreed with this attribute category.

While $66.6 \%$ somewhat agreed, agreed, and strongly agreed that the app's system architecture made it easy to navigate, only $33.3 \%$ agreed and strongly agreed with this usability category, receiving the lowest ranking of all tested areas. This result was consistent with other themes that emerged from the study's interview sessions, where participants expressed concerns with navigation. While $70.4 \%$ somewhat agreed, agreed, and strongly agreed that the app was easy to learn (learnability), only $36.3 \%$ agreed and strongly agreed with this usability category. 
In regard to learnability $90.9 \%$ of those somewhat agreed, agreed, and strongly agreed that the app's command names were easy to learn. However, only $45.5 \%$ of participants somewhat agreed, agreed, and strongly agreed considered the lack of Cancel options to created multiple errors or missteps as they navigated through the system. Finally, regarding the overall satisfaction and userexperience with the mobile app, the score was 6.64 out of 10 .

Open-Ended Interview: Several themes emerged from the outcomes of our interview questions. First. $100 \%$ of participants considered the HYPOalert system has a great value and would positively impact diabetics patients with their daily lifestyle practices, self- managing of their disease, and would help advance their understanding and management of a HYPO condition. A majority of the participants $(92 \%)$ considered the data visualization and color-coded charts with numbers as the best feature, and the one they enjoyed the most in the HYPOalert system. A positive first impression of the system was reported by $85 \%$ of the users. The common themes $(85 \%)$ emerging from the responses regarding the participants' negative experience with the menu options and buttons, i.e., confusion caused difficulties to navigate among different parts of the system. Based on the final interview responses related to frustrating features of the system, $43 \%$ reported navigation, and $14 \%$ reported logbook visuals and BG manual entry processes.

\section{Conclusion}

Hypoglycemia can be a life-threating condition, of which regular metabolic monitoring is critical. There is need for technologies that can help diabetes patients detect and treat HYPO episodes. This is especially true for diabetes patients that suffer from general hypoglycemia or hypoglycemia unawareness, i.e., a rapid drop in $\mathrm{BG}$ without any physiological symptoms. Many traditional methods such as urine tests focus on detecting elevated blood glucose methods, and more modern CGM devices can delay the reporting of HYPO episodes until a patient is too compromised for self-treatment. This paper reported on a two design iterations of a dedicated HYPO monitoring mobile app (HYPOalert), that will accompany a new noninvasive smart sensor that detects HYPO by human breath for Type 1 and 2 diabetics.

After our first design iteration, we interviewed users regarding the system (with static prototypes). Findings suggest a strong reception of HYPOalert. Participants agreed that the proposed system will have a positive impact on managing their HYPO. We then conducted the evaluation of two FDA approved mHealth BG monitoring systems to compare and assess product features. Our comparative analysis was used to inform and enhance the prototype design of the second iteration. We confirmed that customizing BG thresholds for normal. Customization also included setting dangerous levels to enable HYPO alerts and allowing patients to define their own BG self- management plan. Finally, a usability study was used to test the second iteration of the HYPOalert prototype, ease of use and functionality.

The results showed $100 \%$ of participants found the HYPOalert mobile app could have a positive impact on diabetic patients' lifestyle, self-managing of the disease, and daily managing the overall HYPO condition. Study findings also showed $100 \%$ of participants considered the visualization of data and text in the HYPOalert app appealing, and $92 \%$ agreed with the overall interface design. Usability testing results provided significant lessons learned for how to improve the navigation, data visualizations, history logs of $\mathrm{BG}$ level readings/trends, customizable timeframes, and a legend color coding system that accompanies the BG values displayed. The insights captured from the usability study will guide the third design iteration of the actual product, followed by randomized control trial with patients.

\section{Acknowledgements}

This work is supported by a NSF/SCH grant: \#1502310.

\section{References}

[1] Alberti KGMM, Zimmet PZ, (1998) Definition, diagnosis and classification of diabetes mellitus and its complications Part 1: diagnosis and classification of diabetes mellitus Provisional Report of a WHO Consultation. Diab Med 15:539-553.

[2] American Diabetes Association, (1979)Classification and diagnosis of diabetes mellitus and other categories of glucose intolerance. Diabetes28:1039-1057.

[3] American Diabetes Association, (2013) Economic costs of diabetes in the U.S. in 2012. Diabetes Care. 36, 1033 1046 ,

[4] Bergenstal, R. M., Ahmann, A. J., Bailey, T., Beck, R. W., Bissen, J., Buckingham, B., et al. (2013) Recommendations for standardizing glucose reporting and analysis to optimize clinical decision making in diabetes: The ambulatory glucose profile (AGP). Diabetes Technol. Ther. 15, 198-211.

[5] Brazeau, A.-S., Rabasa-Lhoret, R., Strychar, I., and Mircescu, H. (2008) Barriers to physical activity among patients with type 1 diabetes. Diabetes Care 31, 21082109.

[6] Dall, T. M., Mann, S. E., Zhang, Y., Quick, W. W., Seifert, R. F., Martin, J., et al. (2009) Distinguishing the economic costs associated with type 1 and 2 diabetes. Pop. Health Mng. 12, 103-110.

[7] Davey, R. J., Stevens, K., Jones, T. W., and Fournier, P. A. The effect of short-term use of the Guardian RT continuous glucose monitoring system on fear of hypoglycaemia in patients with type 1 diabetes mellitus. Prim. Care Diabetes 6, 35-39.

[8] Elliott, J., and Heller, S. (201q) Hypoglycaemia unawareness. Pract. Diabetes Int. 28, 227-232. 
[9] Maia, F. F. R., and Araújo, L. R. Efficacy of continuous glucose monitoring system (CGMS) to detect postprandial hyperglycemia and unrecognized hypoglycemia in type 1 diabetic patients. (2007). Diabetes Res. Clin. Pract. 75, 30-34.

[10] Munshi, M. N., Segal, A. R., Suhl, E., Staum, E., Desrochers, L., Sternthal, A., et al. (2011). Frequent hypoglycemia among elderly patients with poor glycemic control. Arch. Intern. Med. 171, 362-364.

[11] Oliver, N. S., Toumazou, C., Cass, A. E. G., and Johnston, D. G. (2009) Glucose sensors: A review of current and emerging technology. Diabet. Med. 26, 197-210.

[12] Rachmiel, M., Landau, Z., Boaz, M., Mazor Aronovitch, K., Loewenthal, N., Ben-Ami, M., et al. (2014) The use of continuous glucose monitoring systems in a pediatric population with type 1 diabetes mellitus in real-life settings: The AWeSoMe Study Group experience. Acta Diabetol. .

[13] Rooney, N. J., Morant, S., and Guest, C. (2013) Investigation into the value of trained glycaemia alert dogs to clients with type I diabetes. PLoS ONE 8, e69921.

[14] Stork, A. D. M., van Haeften, T. W., and Veneman, T. F. (2007) The decision not to drive during hypoglycemia in patients with type 1 and type 2 diabetes according to hypoglycemia awareness. Diabetes Care 30, 2822-2826 .

[15] (2008) Wells, D. L., Lawson, S. W., and Siriwardena, A. N. (Canine responses to hypoglycemia in patients with type 1 diabetes. J. Altern. Complement. Med. 14, 12351241 .

[16] Wintergerst, K. A., Buckingham, B., Gandrud, L., Wong, B. J., Kache, S., and Wilson, D. M. (2006) Association of hypoglycemia, hyperglycemia, and glucose variability with morbidity and death in the pediatric intensive care unit. Pediatrics 118, 173-179. 